\title{
On the Four Current Source of the Electromagnetic Fields (*) $\left(^{(*)}\right)$.
}

\author{
K. L. DugGaL (Windsor, Canada)
}

Summary. - In this paper, we consider the field equations of Einstein-Maxwell theory in the presence of a four-current souree $J$ with vanishing Lorentz force. By relating $J$ with one of the eigenvatues of the contracted curvature tensor, we discuss some of its properties. In particular, we find certain geometric conditions governing the direction of $J$. Physically, the results of this paper may be applicable in the study of the history traced by $J$ and in the theory of geometrodynamics.

\section{1. - Prerequisities.}

Consider the 4-dimensional space-time manifold $V_{4}$ of general relativity, with the gravitational symmetrie tensor $h$ of signature $(+++-)$. Let ' $F$ ' be the electromagnetic tensor field (skew-symmetrie) of type $(0,2)$. Using $h$, one can get a tensor field $F$ of type $(1,1)$ defined by

$$
h(F X, X)={ }^{\prime} F(X, Y),
$$

where $X$ and $Y$ are arbitrary vector fields. According to HLAVATY ${ }^{\prime}[6], F$ admits two real invariant $K$ and $k$ defined by

$$
4 K=\operatorname{trace}\left(F^{2}\right), \quad k=\operatorname{det}(F), \quad D=K^{2}-k .
$$

For the non-null field there exists a frame $R=\left\{e_{a}\right\}$ of null vectors $(1 \leqslant a, b \leqslant 4)$ at each point of $V_{4}$ such that $e_{1}, e_{2}$ are conjugate complex and $e_{3}, e_{4}$ are real. Their corresponding eigenvalues are $\{i P,-i P, Q,-Q\}$, where

$$
P=\sqrt{\sqrt{D}+K}, \quad Q=\sqrt{\sqrt{D}-K} .
$$

For the second class of $F$, det $(F)=0$ and therefore

$$
P=0, \quad Q=\sqrt{-2 K}, \quad K<0,
$$

or

$$
P=\sqrt{2 K}, \quad Q=0, \quad K>0
$$

(*) Entrata in Redazione il 26 agosto 1978.

(**) This research was supported by the National Research Council of Canada.

20 - Annali di Matematica 
For null $F$, it is possible to use the same frame $R$ with the understanding that $e_{4}$ is the only eigen vector of $F$ having eigen value zero. The Maxwell equations in the presence of a four current source $J$ may, with a suitable choice of units be written in the form:

$$
\operatorname{div} F=J ; \quad d F=0
$$

where $d$ is the operator of exterior derivative and $J$ satisfies the continuity equation

$$
\operatorname{div} J=0 \text {. }
$$

$J$, in general, can be expressed as

$$
J=\Theta e_{1}+\bar{\Theta}_{e_{2}}+\beta e_{3}+\gamma e_{4}
$$

where $\Theta, \bar{\Theta}$ are conjugate complex and $\beta, \gamma$ are real source scalars. The basic system of Einstein's field equations can be written in the form [6]

$$
R X=\mu \eta(X) \xi+E X+\left(p+\frac{1}{2} R\right) X,
$$

where $\mu, p, R X$ and $R$ are the density, the pressure, the contracted eurvature and scalar curvature respectively, $\xi$ is the time-like velocity vector of its orbit and $E X$ is the electromagnetic energy tensor of type $(1,1)$ defined by

$$
E X=F^{2} X+K X
$$

Contracting (3a), we get

$$
\mu=R+4 p
$$

Let us set

$$
\alpha \stackrel{\text { def }}{=} \frac{1}{2} R-\sqrt{D}+p
$$

Throughout this investigation, we assume that the Lorentz force vanishes which means that $F J=0$. Now $F J=0$ would admit a nonzero solution iff $\operatorname{det}\left(F^{\prime}\right)=0$ which implies that the non-zero $F$ belongs only to the second class.

\section{2. - Non-null $F$.}

In this section, we assume that $\mu>0$ and without any loss of generality

$$
h(X, Y)=e^{1}(X) e^{2}(X)+e^{2}(X) e^{1}(Y)+e^{3}(X) e^{4}(Y)+e^{4}(X) e^{3}(X),
$$


where $\left\{e^{a}\right\}$ is the dual of $\mathcal{R}$. Thus, we have

$$
J \cdot J=2(\Theta \bar{\Theta}+\beta \gamma) .
$$

Operating $F$ to $(2 e)$ and using $F J=0$, we state

Propostition 1. - A non-null electromagnetic field with a four current source and vanishing Lorentz force must have the following relations of the source sealars:

(a) $K<0: \Theta \neq 0, \beta=\gamma=0$,

(b) $K>0: \Theta=0, \beta$ and $\gamma$ arbitrary but both not zero.

Corollary 1. $K<0$ implies that $J$ is always space-like.

(i) Case $K<0$ : For this case, it has been shown in [2] that, corresponding to two complementary distributions $L$ and $\tilde{L}$, ths space-time $V_{4}$ can be endowed with a geometric structure, called almost contingent metric structure, $(F, U, V, u, v$, $h, K<0)$ defined by

$$
\begin{aligned}
& F^{2} X+2 K X-2 K\{u(X) U+v(X) V\}=0, \\
& F U=F V=0, \quad u(U)=v(V)=1, \quad u(V)=v(U)=0,
\end{aligned}
$$

where $\{U, V\}$ spans $L,\{u, v\}$ is its dual and $\sqrt{D}=-K$. Furthermore, the conjugate complex frame $\mathcal{R}$ can be locally replaced by a real frame $R^{*}=\left\{U, V, e_{3}, e_{4}\right\}$ such that

$$
e_{1}=\frac{U-i V}{\sqrt{2}}, \quad e_{2}=\frac{U+i V}{\sqrt{2}},
$$

and the metric $h$ satisfies the following relations:

$$
\begin{aligned}
& h(U, U)=h(V, V)=1, \\
& h(U, X)=u(X), \quad h(V, X)=v(X) .
\end{aligned}
$$

Expressing $J, E X$ and $R X$ locally in terms of the transformed real frame $R^{*}$, we get

$$
\begin{aligned}
J & =\sqrt{2}(\operatorname{Re} \Theta U+\operatorname{Im} \Theta V) \\
E X & =K\left(u(X) U+v(X) V-e^{3}(X) e_{3}-e^{4}(X) e_{4}\right) \\
R X & =\alpha(u(X) U+v(X) V) \\
& +(\alpha-2 K)\left(e^{3}(X) e_{3}+e^{4}(X) e_{4}\right) \\
& -\frac{\mu}{\Theta \bar{\Theta}}(\operatorname{Re} \Theta u(X)+\operatorname{Im} \Theta v(X))(\operatorname{Re} \Theta U+\operatorname{Im} \Theta V),
\end{aligned}
$$


where we have set $J=\mu \xi$. Putting $U, V$ for $X$ in $(6 c)$, we get

$$
\begin{aligned}
& R U=\left(\alpha-\frac{\mu}{\Theta \bar{\Theta}}(\operatorname{Re} \Theta)^{2}\right) U-\left(\frac{\mu}{\Theta \bar{\Theta}} \operatorname{Re} \Theta \operatorname{Im} \Theta\right) V \\
& \left.R V=\left(\alpha-\frac{\mu}{\Theta \bar{\Theta}} \operatorname{Im} \Theta\right)^{2}\right) \nabla-\left(\frac{\mu}{\Theta \bar{\Theta}} \operatorname{Re} \Theta \operatorname{Im} \Theta\right) U .
\end{aligned}
$$

It is well-known [6] that $\alpha$, as an eigenvalue of $R X$, plays an important role in the study of geometrodynamics. Now, it is clear from (7ab) that $\alpha$ is an eigenvalue of $R X$ with eigenvector $U$ or $V$ iff $\operatorname{Re} \Theta=0$ or $\operatorname{Im} \Theta=0$ which further implies from (6a) that iff $J$ moves parallel to $V$ or $U$ respectively. This leads to the following theorem.

THEOREM 1. - Let the space-time $\nabla_{4}$ be endowed with an almost contingent metric structure $(F, U, V, u, v, h, K<0)$ defined by all the relations of (5). Then, the four current source $J$ with vanishing Lorentz force moves parallel to $V$ or $U$ iff $\alpha$ is an eigenvalue of the contracted curvature tensor $(\mu>0)$ with eigenvector $U$ or $V$ respectively.

It has been proved in [4] that under certain geometric conditions $U$ and $V$ are Killing vectors which are symmetries of ' $F$. Also, it is known [11] that a Killing vector $X$ attains the velocity of light iff $R X=0$. Although in general, $U$ and $V$ are space-like vectors of norm $\sigma$ ( $c^{\infty}$ function) but, in particular, any one of them can become null and, thereby, attain the velocity of light. Such Killing vectors with light velocity generate null hypersurfaces, called. Killing horizons [7].

CORoLlaRY 2. - The eigenvalne $\alpha$ of the contracted curvature tensor $(\mu>0)$ is zero iff $U$ or $V$ (but noth both) is Killing vector with light velocity.

It can easily be deduced from the result (2.8)b of [6] that the basic system consisting of $(2 a)$ and $(3 a)$ is compatible with the continuity equation $(2 b)$ if $p$ is constant along the $J$-curves i.e. if

$$
J(p)=0
$$

In order to examine above compatibility condition with respect to $(F, U, V, u, v, h$, $K<0)$ structure, we substitute $J$ from $(6 a)$ and get

$$
\operatorname{Re} \Theta U(p)+\operatorname{Im} \Theta V(p)=0
$$

Applying the results of theorem 1 in $(8 b)$, we state

Corollary 3. - The necessary and sufficient condition for the compatibility of the basic system $(2 a)$ and $(3 a)$ with the continuity equation $(2 b)$ is that the pressure $p$ is constant along the $U$-curves or the $V$-curves according as $\alpha$ is an eigenvalue with eigenvector $V$ or $U$ of the contracted curvature tensor $(\mu>0)$. 
Furthermore, it follows from corollaries 2 and 3 that the basic system $(2 a)$ and $(3 a)$ is compatible with the continuity equation (2b) iff the pressure $p$ is constant along the $V$-curves or $U$-curves according as $U$ or $V$ is Killing with light velocity. This last result is consistent with the theorem 2 of [3].

(ii) Oase $K>0$. For this case, it has been shown in [2] that, corresponding to two complementary distributions $L$ and $\tilde{L}$, the space-time $V_{4}$ can be endowed with a geometric structure, called almost contingent ( $\left.{ }^{1}\right)$ metric structure, $(F, \tilde{U}, \tilde{V}$, $\tilde{u}, \tilde{v}, h, K>0$ ) defined by

$$
\begin{aligned}
& F^{2} X+2 K X-2 K\{\tilde{n}(X) \tilde{U}+\tilde{v}(X) \tilde{V}\}=0, \\
& F \tilde{U}=F \tilde{V}=0, \quad \tilde{u}(\tilde{U})=\tilde{v}(\tilde{V})=1, \quad u(V)=v(U)=0,
\end{aligned}
$$

where $\{\tilde{U}, \tilde{V}\}$ spans $\tilde{L},\{\tilde{u}, \tilde{v}\}$ is its dual set and $\sqrt{D}=K$. Furthermore, the frame $\mathcal{R}$ can be locally replaced by another frame $\tilde{R}=\left\{e_{1}, e_{2}, \tilde{U}, \tilde{V}\right\}$ such that

$$
e_{3}=\frac{\tilde{U}+\tilde{V}}{\sqrt{2}}, \quad e_{4}=\frac{\tilde{U}-\tilde{V}}{\sqrt{2}}
$$

and the metric $h$ satisfies the following relations

$$
h(\tilde{U}, \tilde{U})=h(\tilde{V}, \tilde{V})=1, \quad h(\tilde{U}, X)=\tilde{u}(X), \quad h(\tilde{V}, X)=-\tilde{v}(X)
$$

Expressing $J, E X$ and $R X$ locally in terms of the transformed frame $\tilde{\mathcal{R}}$ we get

$$
\begin{aligned}
J= & \varphi \tilde{U}+\psi \tilde{V}, \quad \varphi=\frac{\beta+\gamma}{\sqrt{2}}, \quad \psi=\frac{\beta-\gamma}{\sqrt{2}}, \\
E X & =K\left(\tilde{u}(X) \tilde{U}+\tilde{v}(X) \tilde{V}-e^{1}(X) e_{1}-e^{2}(X) e_{2}\right), \\
R X & =\alpha(\tilde{u}(X) \tilde{U}+\tilde{v}(X) \tilde{V})+(\alpha-2 K)\left(e^{1}(X) e_{1}+e^{2}(X) e_{2}\right) \\
& -\frac{\mu}{2 \beta \gamma}(\varphi \tilde{u}(X)-\psi \tilde{v}(X))(\varphi \tilde{U}+\psi \tilde{V}),
\end{aligned}
$$

where we have again set $J=\mu \xi$. Putting $\tilde{U}, \tilde{V}$ for $X$ in (10c), we get

$$
\begin{aligned}
& R \tilde{U}=\left(\alpha-\frac{\mu}{2 \beta \gamma} \varphi^{2}\right) \tilde{U}-\frac{\mu \varphi \psi}{2 \beta \gamma} \tilde{V}, \\
& R \tilde{V}=\left(\alpha+\frac{\mu}{2 \beta \gamma} \psi^{2}\right) \tilde{V}+\frac{\mu \varphi \psi}{2 \beta \gamma} \tilde{U} .
\end{aligned}
$$

(1) As almost contingent structure of second class is not unique, in this paper, we adjust such that the local transformation satisfies $(9 b), \tilde{U}$ and $\tilde{V}$ are real and $\tilde{V}$ is time-like. 
Now, it is clear from $(10 d, e)$ that $\alpha$ is an eigenvalue of the contracted curvature tensor $(\mu>0)$ with eigenvector $\tilde{U}$ or $\tilde{V}$ iff $\varphi=0$ or $\psi=0$ respectively. This leads to the following theorem.

THEOREM 2. - Let the space-time $V_{4}$ be endowed with an almost contingent metrie structure $(F, \tilde{U}, \tilde{u}, \tilde{v}, h, K>0)$ defined by all the relations of (9). Then, the four current source $J$ with vanishing Lorentz force moves parallel to $\tilde{V}$ or $\tilde{U}$ if $\alpha$ is an eigenvalue of the contracted curvature tensor $(\mu>0)$ with eigenvector $\tilde{U}$ or $\tilde{V}$ respectively.

Proceeding exactly as in case $K<0$, we state the following corollaries.

Corollarr 4. - The eigenvalue $\alpha$ of the contracted curvature tensor $(\mu>0)$ is zero iff $\tilde{U}$ or $\tilde{V}$ (but not both) is Killing vector with light velocity.

COROLlaRY 5. - The necessary and sufficient condition for compatibility of the basic system $(2 a)$ and $(3 a)$ with the continuity equation $(2 b)$ is that the pressure $p$ is constant along the $\tilde{U}$-curves or the $\tilde{V}$-curves according as $\alpha$ is an eigenvalue with eigenvector $\tilde{V}$ or $\tilde{U}$ of the contracted curvature tensor $(\mu>0)$.

REMARK 1. - Hlavaty' [6] studied one of the fundamental problems of expressing the physical data $\mu, \xi E X$ and $p$ as algebraic concomitants of the contracted curvature tensor $R X(\mu>0)$ for the non-null electromagnetic fields. According to his study, a solution exists iff $R X$ has one of the four characteristics [1111], [112], [(11), 11], $[(111), 1]$ and if the corresponding eigenvalues satisfy certain inequalities. Throughout that discussion of Hlavaty', $\alpha$ as an eigenvalue of $R X$ played a major role in finding the conditions under which the four eigenvalues exist for all the four characteristics. In this paper, we have extended the results of Hlavaty' by relating $\alpha$ with the four current source $J$. In particular, we have found certain geometric conditions governing the direction of $J$. Physically, such information may be useful in problems related to the history traced by $J$ and to the theory of geometrodynamics.

REMARK 2. - The results presented in this section may also be useful in the study of null stationary hypersurfaces which are called Killing horizons [7].

\section{3. - Null $F$.}

In this section we assume without any loss of generality

$$
h(X, Y)=e^{1}(X) e^{2}(X)+e^{2}(X) e^{1}(Y)-e^{3}(X) e^{4}(Y)-e^{4}(X) e^{3}(X) .
$$

Thus the following will hold.

$$
\begin{aligned}
& \sqrt{2} F X=e^{3}(X)\left(e_{1}+e_{2}\right)+\left(e^{1}(X)+e^{2}(X)\right) e_{4}, \\
& \sqrt{2} F e_{1}=\sqrt{2} F e_{2}=e_{4}, \quad \sqrt{2} F e_{3}=e_{1}+e_{2},
\end{aligned}
$$


(11c)

$$
\begin{aligned}
& F^{2} X=e^{3}(X) e_{4}, \quad F e_{4}=0, \quad e^{3} \cdot F=0 . \\
& J \cdot J=2(\Theta \bar{\Theta}-\beta \gamma) .
\end{aligned}
$$

Operating $F$ to $(2 c)$, using $(11 b)$ and $F J=0$, we state

Proposition 2, - A null electromagnetic field with a four current source and vanishing Lorentz force must have the following relations of the source scalars:

$$
\Theta+\bar{\Theta}=0, \quad \beta=0
$$

CORoltary 6. - A null electromagnetic field with vanishing Lorentz force can not admit a time-like current source.

Expressing $J$ locally in terms of the frame $R$ and using above results, we state

CoRollaRY 7. - For a null electromagnetic field with vanishing Lorentz force, the four current source $J$ can be expressed as:

(c) $J$ space-like: $J=\Theta\left(e_{1}-e_{2}\right)+\gamma e_{4}$,

$$
\text { (d) } J \text { null: } \quad J=\gamma e_{4} \text {. }
$$

(iii) $J$ space-like. This means that $\Theta \neq 0$. Expressing $R X$ locally in terms of the frame $R$, we get

$$
R X=\alpha X+e^{3}(X) e_{4}-\frac{\mu}{2 \Theta^{2}}\left(\Theta e^{2}(X)-\Theta e^{1}(X)-\gamma e^{3}(X)\right)\left(\Theta e_{1}-\Theta e_{2}+\gamma e_{4}\right),
$$

where we set $J=\mu \xi, \mu>0$ and $\alpha=\frac{1}{2} R+p$.

Definition. - A vector $T$ is called polarisation vector of null $F$ if

$$
\sqrt{2} T=e_{1}+e_{2}, \quad \sqrt{2} t=e^{1}+e^{2} .
$$

It is easy to verify that $T$ will satisfy the following relations:

$$
\begin{aligned}
& F T=e_{4}, \quad t \cdot F=e^{3}, \quad t(T)=1, \\
& e^{1}(T)=e^{2}(T)=\frac{1}{\sqrt{2}}, \quad e^{3}(T)=e^{1}(T)=0 .
\end{aligned}
$$

Putting $T$ and $e_{4}$ in $(13 a)$, we get

$$
R T=\alpha T, \quad R e_{4}=\alpha a_{4} .
$$


It is important to note that $(13 d)$ also holds for $\mu=0$. Let $S$ be the eigenspace generated by the eigenvectors $T$ and $e_{4}$ of $R X$ with eigenvalue $\alpha$. This leads to the following theorem:

Theorem 3. - The field equations of a null electromagnetic field, with space-like four current source $J$ and vanishing Lorentz force, are compatible when $\mu \geqslant 0$ if $J$ belongs to the eigenspace $S$ generated by the eigenvectors $T$ and $e_{4}$ both having eigen value $\alpha$.

Mishra [8], while studying some problems of geometrodynamics, has expressed the physical data $\mu, \xi, E X, p$ explicitly as algebraic concomitants of $R X$ for the null electromagnetic fields. According to his study, the field equations are consistent iff $\alpha$ is a double eigen value of $R X$ whose characteristic is [(11)11] when $\mu>0$ and [(211)] when $\mu=0$. Again, we have extended the results of Mishra by relating $\alpha$ with the four current source $J$. Following results are easily verified.

Corollary 8. - A necessary condition that the field equations are compatible when $\mu \geqslant 0$ is that $\alpha$ is only a double eigenvalue.

It is known [8] that the basic system consisting of $(2 a)$ and $(3 a)$ is compatible with the continuity equation if $p$ is constant along the $\xi$-curves i.e. $\xi(p)=0$. If $\mu=0$, then $(3 e)$ implies that $R+4 p=0$. Thus, substituting $p=-\frac{1}{4} R$ in $\xi(p)=0$, we state:

Proposition 3. - The field equations are compatible when $\mu=0$ if the scalar curvature $R$ of space-time is constant along $\xi$-curves.

The results of corollary 8 and proposition 3 have been proved in [8] by a different method.

(iv) $J$ Null. This means that $\Theta=0$ and, therefore,

$$
J=\gamma e_{1} .
$$

At this point, the following two observations are worth mentioning:

1) $J$ is a real source for a null electromagnetic field.

2) $J$ is fictitious and does not. generate the null electromagnetic field.

ZUND [12] has tried to justify above mentioned behavior of null source by discussing the two celebrated GoLdBerg-KerR [5] and Mariot-Robinson [9, 10] theorems. According to his viewpoint, the null source either plays the role of a "boost" for the radiation field or merely remains passive and not affect the field. In support of this, he has proved that the presence of the null source does not modify the conclusions of the Mariot-Robinson theorem for source free fields. In the same paper, Zund has further referred to Bateman's pioneering work on the electromagnetic theory of lightdarts [1] where a null source is called a light-dart. In view of vital physical impor- 
tance of Bateman's work, we reproduce some of his remarks. He states that "These solutions show that light-darts of an electrical nature may have a real existence and that it is not impossible for electricity to travel with the velocity of light. The chief difficulty in developing an electromagnetic theory of light-darts is that of the location of the energy of a light-dart. It is not certain that we can speak of a definite location of energy. Furthermore, he concludes that "There may, of course, be a difference of opinion as to the physical interpretation of these results. A point of view that seems fairly reasonable is that the energy calculated is really in the field when the lightdart produces a field, but is in the light-dart when the light-dart fails to produce a field owing to the absence of aether-particles in the immediate neighbourhood of the primary source when the light-dart is being emitted from it ${ }^{\circ}$.

\section{REFERENCES}

[1] H. Batemax, An electromagnetic theory of lighi-darts, Bull. Amer, Math. Soc., 29 (1923), pp. 385-393.

[2] K. L. DUGGaI, Geometry developed by the electromagnetic tensor field, Ann. di Mat. pura e appl., (IV), 119 (1979), pp. 239-245.

[3] K. L. DUGGAL, Einstein-Maxwell equalions compatible with certain Killing vectors with light-velocity, Ann. di Mat. pura e appl., (IV), 120 (1979), pp. 263-268.

[4] K. L. DUGGaL, Existence of two Killing vector fields on the space-time of general relativity, tensor, N. S., 32 (1978), pp. 318-322.

[5] J. N. GoldBenG - R. P. KenR, Asymptotic properties of the electromagnetic field, J. Math. Phys., 5 (1964), pp. 172-176.

[6] V. Hravaty', Contributions to the theory of general geometrodynamios, Ann. di Mat. pura e appl., 61 (1963), pp. 121-149.

[7] S. W. Hawkins - G. F. R. Extrs, The large scale structure of spacetime, Cambridge University Press (1973).

[8] R. S. Mishra, Einstein-Maxwell field equations, Third class, Rend. Circ. Matem. Palermo, 11 (1962), pp. 157-177.

[9] L. MaRIot, Le champ éleotromagnétique pur en relativité générale, Thèse, Paris (1957).

[10] I. Rosinson, Null electromagnetic fields, J. Math. Phys., 2 (1961), pp. 290-291.

[11] K. Yavo, Integral formulae in Riemannian geometry, Marcel Dekker Inc. (1970).

[12] J. D. ZuND: Electromagnetic theory in general relativity. - IV: A theory of light-darts, Tensor, N. S., 28 (1974), pp. $283-289$. 